\title{
A new solution for the Energy Packet-based Dispatching using power/signal dual modulation
}

\author{
Edoardo De Din \\ Institute for Automation of Complex Power Systems - \\ RWTH Aachen University \\ Aachen, Germany \\ ededin@eonerc.rwth-aachen.de \\ Veit Hagenmeyer \\ Institute for Applied Informatics - Karlsruhe Institute of \\ Technology \\ Karlsruhe, Germany \\ veit.hagenmeyer@kit.edu
}

\author{
Antonello Monti \\ Institute for Automation of Complex Power Systems - \\ RWTH Aachen University \\ Aachen, Germany \\ amonti@eonerc.rwth-aachen.de \\ Klaus Wehrle \\ Chair of Communication and Distributed Systems - \\ RWTH Aachen University \\ Aachen, Germany \\ wehrle@comsys.rwth-aachen.de
}

\begin{abstract}
The current process of transformation affecting the energy system is calling for completely new approaches. This paper investigates the possibility of introducing energy packets as a fundamentally new abstraction concept that is following the transformation of communication networks in the 1970s from synchronous circuit switching to asynchronous store-and-forward routing. Focus is placed on the exploitation of power electronic devices in creating information embedded into the power flow. Considerations on some criticalities and future developments that are needed to further proceed on the development of this new vision are discussed. A simple test in Matlab/SIMULINK is provided in support for the packet dispatching described in the paper.
\end{abstract}

\section{KEYWORDS}

Energy Packet, Signal Transmission, Power Dispatching

\section{ACM Reference Format:}

Edoardo De Din, Antonello Monti, Veit Hagenmeyer, and Klaus Wehrle. 2018 A new solution for the Energy Packet-based Dispatching using power/signal dual modulation. In e-Energy '18: The Ninth International Conference on Future Energy Systems, June 12-15, 2018, Karlsruhe, Germany. ACM, New York, NY, USA, 5 pages. https://doi.org/10.1145/3208903.3208931

\section{INTRODUCTION}

The Energy Transformation, the so called Energiewende in German language, is challenging all the main principles around which the electrical infrastructure has been operated for a long time. In particular, the growing role played by volatile energy sources, e.g. wind and sun, is challenging the traditional grid frequency control based on an instantaneous balance between generation and load [8]. So far, the system has operated as load driven: loads represent the requirement and generation is adapted accordingly. Thus it is



This work is licensed under a Creative Commons Attribution-NonCommercial International 4.0 License.

e-Energy '18, June 12-15, 2018, Karlsruhe, Germany

(c) 2018 Copyright held by the owner/author(s).

ACM ISBN 978-1-4503-5767-8/18/06.

https://doi.org/10.1145/3208903.3208931 necessary to consider completely new concepts able to remove the requirement of instantaneous balance over the complete infrastructure: in other words, a possible approach to the solution is given by the option to remove the condition of synchronicity between generation and consumption. In effect, it is possible to get inspiration from a similar process that has affected the communication field. Considering history, the traditional telephone network used to have a hierarchical structure and characteristics - in terms of limited reliability, flexibility and capacity - that are comparable to today's energy infrastructure. Key concepts to achieve these characteristics are a self-organized decentralized organization and the utilization of packets that travel asynchronously through the network on a packet-by-packet basis instead of a time-synchronous reservation transmission time slots. Another important feature of a packet-based solution is the paradigm of store-and-forward-routing. Such a solution enables a timely decoupling of the used transmission resources. Current electrical grids can be seen - similar to circuit-switching technology in telecommunication - as a synchronous coupling and fixed allocation of connections and resources, whereas store-and-forward-routing enables a more asynchronous coupling of connections and a statistical usage of resources, since the autonomous packets can be delayed by short-term buffering. The growing presence of storage options in the energy system [3] [11] brings to consideration that similar ideas of store-andforward-routing could benefit the energy infra-structure in two main ways: (i) creating a clear decoupling between demand and generation; (ii) allowing always best use of the infrastructure capacity. Furthermore, looking at the current energy system, two more considerations support this vision: (i) at user level, many components operate in a way that is naturally close to a packet concept such as batteries or heating systems; (ii) at market level, today's trading is already based on quantized amounts of energy. In the present paper, the authors, starting from this vision, analyze how a concept of abstraction of energy packet can be developed and implemented in the electrical infrastructure. Main focus is then placed on the possibility to embed information in the energy flow, so to mimic the idea of an information packet. Some simulation results are also used to show the feasibility of the proposed approach. 


\section{INTERNET AND INTERNET PROTOCOL AS A FOUNDATIONAL RESEARCH BRIDGE}

As stated in the Introduction, the vision behind the present paper is the transformation of the energy system into a new level of abstraction that would allow the elements of the system to be managed in the same manner as the elements of a computer network. The foundation to achieve this abstraction procedure is the definition of the Energy Packet that represents the equivalent of the data packet in the Internet Protocol (IP).

Therefore the new concept should follow the fundamentals of the internet architecture that describes the procedure for packet routing and the rules for the encapsulation of the data within the IP - the so-called "in-band" signaling [14]. Similarly, the quantized packet of energy to be dispatched to a destination host should be encapsulated into blocks of energy. Moreover, to perform a correct routing, the different elements connected to the electrical grid should have an address in the same way as the hosts in a computer network. In any case is it clear that the dispatching of the Energy Packets through the electrical grid needs a communication system to associate a certain information to the quantized energy. Therefore the implementation of the proposed vision could make use of power electronic converters, because they interface the Distributed Energy Resources (DERs), the Energy Storage Systems (ESSs) and the loads with the electric grid and they can be used to superimpose an information signal to the power signal [16]. In an Energy Packet-based network there can be a smaller grid (a local network), for example an interconnection of several households, where all the elements interfaced with a power converter exchange energy packets, and a larger grid, the distribution grid managed by the Distribution System Operator (DSO), that considers the smaller grid as an aggregation of loads and generator. The two network areas would possess different addresses assignment and the interconnection would be realized by means of an interfacing power converter. This converter acts similarly as the Internet Gateway in the IP network that forwards the data packets to another network.

In the same way as the IP is part of the Open System Interconnection (OSI) model, the packet dispatching can represent only part of a more complex view, as described in [10]. Following this approach, the Energy Packet would perform the abstraction of the devices that physically transmit the data. This permits the application of the solutions already developed for the computer network, given that the Energy Packet would allow to see the electrical grid from an hardware-independent layer. For this reason, at this first stage, the key aspect to enhance the fulfilment of an electrical grid based on the concept of the Energy Packets is the realization of a system for the local dispatching and then later in a wider area. Therefore it is necessary to investigate possible structures for the electrical grid, to define the message and find a suitable method for routing the packets. All these aspects are covered in Section 4, Section 5 and Section 6.

\section{A CONCEPT OF ENERGY PACKET}

An Energy Packet (EP) is a defined amount of energy that can be stored and routed through any kind of energy network. This idea is inspired from the Internet Technology (discrete and autonomous entities: IP packets). When it comes to electrical networks, an EP implies the possibility to route a specific amount of energy by properly shaping the current flow and supporting energy routing. Power electronics make the first requirement extremely easy to achieve and in the literature of this area already several examples of energy routers are presented: For example in [4], a complete architecture based on Energy Packet is proposed as possible approach to energy distribution. In [13] and [12], viceversa, practical solutions for the implementation of energy routers are proposed. As already introduced in [10], the IP approach used in communication networks can inspire a complete restructuring of the electrical energy system. Following the definition at the beginning of the section, some key characteristics emerge:

- the focus is more on energy than power

- the amount of energy is pre-defined as a quantized quantity. Different definitions of the quantization are acceptable within the same infrastructure (for example different voltage levels may work with a different quantization definition)

- the energy dispatch is associated with an information packet which can be used for different purposes. A typical element of information would be an address supporting the dispatch of an Energy Packet to a specific destination.

A second critical element of the Internet approach is the idea of store-and-forward routing. This operation is performed by the routers and implies that each information package is shortly stored in the node, and then forwarded in the best moment through the best momentarily route. The energy router provides the possibility to actively control the power flow and it also introduces a decoupling feature in the infrastructure, so that energy and information at the same time do not flow "freely" through the infrastructure. This consideration has very important consequences. As the Internet itself is a "network of networks", a combination of very different local and long-distance networks, the overall system becomes the integration of numerous local systems. There is consequently also no need for a central intelligence because the system is simply not operating as a globally coordinated infrastructure.

\section{STRUCTURE OF THE LOCAL DISPATCHING NETWORK}

The dispatching network describes the grid arrangement, the power converters and the control structure that are necessary for energy packet generation and dispatching in a local area.

An original solution for the generation and dispatches of energy packets (called "power packets" in the papers) is described in [13] [12] and supported by experimental results. The authors have presented a new system for energy dispatching based on the definition of Mixer and Router. The Mixer is a power electronic device that collects the sources and dispatch the energy packet. The Router stores the received energy packet and dispatches it towards the assigned loads. This configuration identifies a complete new structure of the electrical grid that would lead to some difficulties in the implementation on the current grid configuration. Furthermore, the multiple connection of the mixers is prohibited [13].

Recently some studies have performed data modulation by means of PWM strategy on DC power converters [16] [5] [15]. Although the papers have not focused specifically on the concept of the energy packets, they investigated possible structures of the DC 


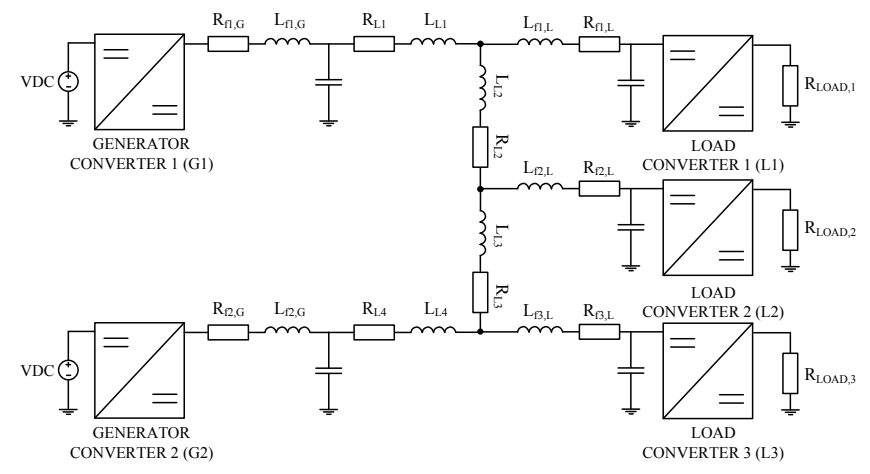

Figure 1: LVDC Grid

grid that would allow the communication among power converters. All the structures comprehend a common DC bus to which all the generation-side converters and the load-side converters are connected, as it is also described in [6]. This grid configuration, which will be used in the simulation example, is described in Fig. 1.

\section{DESCRIPTION OF THE MESSAGE}

After defining some possible structures to achieve the packet dispatching, a specific attention is paid to the information that has to be sent as part of the packet itself. As stated in Section 2, this represents one of the main analogies with the computer network technologies and the key aspect that has to be investigated to enhance this new vision. As described in [2] [13] and in [10], the main element of the information attached to the energy packet is the address, which supports the dispatch of the energy packet to the specific destination. Moreover, some other information could be added in order to exchange additional data between the sender and the receiver such as length of the packet and other possible useful information for the receiver - increasing, however, the complexity of the message itself.

The authors in [2] propose the use of the powerline communication (PLC) for the data communication among all the elements of the digital grid. However, although PLC represents a mature communication technology, it requires independent infrastructure for the signal injection, the amplification and the signal processing. Moreover, the large capacitance of the main dc-bus requires a relatively high-power coupling circuit [5].

Differently from the strategy described [13], a method for modulating signal and power has been proposed in [16] [5] [15]. With this approach the message is superimposed on the power signal by modulating the pulsewidth modulation (PWM) technique of the power converter. This results in a power converter that realizes a power line communication (PLC) [9] acting as a PLC modem. The fundamentals of these modulation strategies for the packet dispatching are described in Section 6. The coding implementation could eventually make use of the media access control (MAC) layer, as suggested in [5], which is described in the the IEEE standard for PLC [1].

\section{PSMD TECHNIQUES FOR PACKET DISPATCHING}

As described in Section 4 and Section 5, a power/signal dual modulation (PSDM) has been recently developed to integrate signal transmission with power conversion. These modulation techniques have been described extensively for PLC systems applications in [9] [1] and some of them have been implemented for PSDM. The first technique that has been applied is the frequency-shift key (PWM/FSK) modulation, where the PWM carrier is switched between two different frequencies to generate the logic binary signal [16].

This PWM/FSK exhibits some issues related to the modulation technique that appears also on PLC [9]. The FSK modulation based on two carrier frequencies does not use the bandwidth in an efficient way and is susceptible to disturbances, meaning that without a control error method a reliable communication is not possible [9]. Moreover, there are some problems that refer to the application of the FSK to the DC-DC converters. Other research activities seem to be necessary to improve the PWM/FSK technique, such as the implementation of a MAC protocol for the multiple node communication and multiple FSK.

Another single carrier modulation strategy is the phase-shift key (PWM/PSK), in which the data transmission is obtained by introducing a $180^{\circ}$ phase shift on the PWM carrier. This method does not modify the switching frequency of the converter and, in addition with a spread spectrum technology (SST), can reduce the EMI emission [15]. The generation of the direct sequence spread spectrum (DSSS) PWM/PSK is based on data spreading and power and data modulation. The data spreading consists on defining a binary pseudo-random sequence (BPRS), i.e. the m-sequence, whose elements are called "chips" that are multiplied with the information signal. The resulting signal, with a spread spectrum, is used to modulate the PWM carrier. The decoding procedure follows exactly the same approach, applying the data demodulation and the $\mathrm{m}$ sequence to the received signal.

This method is used in Section 7 to realize an energy packet dispatch based on PSMD. The key elements to transmit the signals to the specific receivers are the $m$-sequences: each receiver owns a different orthogonal spreading code. Therefore, the DC-DC converter that intends to send a message to a certain receiver (a particular load connected to the same dc bus) uses the specific msequence of the receiver, given that for the others the signal results in a non-decodable message. To perform a packet dispatching, the sender can send a message to the receiver that contains information to arrange the energy packet transmission. If the receiver answers with a positive message, the sender starts sending the energy packet. This simple negotiation procedure exhibits a similarity with the handshaking process in network communication [14]. During the transmission of the energy packet, the sender can keep sending messages to eventually inform the receiver that the conditions has changed, e.g. when the sender is not able to provide any more the same amount of power. 



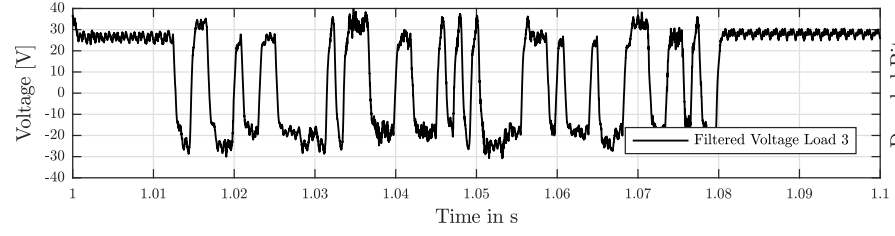

(a) Filtered Voltages



(b) Decoded Messages

Figure 2: Results of the simulation test

\section{SIMULATION EXAMPLE}

The intention of the present simulation example is to describe the application of the PSMD technique for the energy packet generation and dispatch. The simulated system is a low voltage (LV) DC circuit composed by a main bus where all the loads and generators are connected [7] [10]. All the tests have been performed in Matlab/SIMULINK environment with SimPowerSystem toolbox. Both the generation-side and load-side converters are realized with synchronous DC-DC buck converters, where the former regulates the voltage by stepping down the voltage of the DC source whereas the latter controls the current absorbed by the load.

The simulation consists in testing the multinode communication between generators and loads based on DSSS PWM/PSK technique. Fig. 2a shows the filtered measured voltages and Fig. $2 \mathrm{~b}$ the messages decoded by the three loads. Load 1 and Load 2 have received the message from one generation-side converter (see Fig. 1), whereas Load 3 has received the message from a different one. As it appears clearly from the figures, Load 2 exhibits the worst decoding because, being closer to both generation-side converters, it suffers more from the disturbances. This example shows also the importance of synchronizing the operation of sending and decoding, because the receivers need to synchronize the decoding procedure with the superimposed voltage signal. Therefore one way to synchronize the overall system is to use a common synchronizing signal and regulate the operation of sending messages with it, as it has been described in Section 6.

\section{CONCLUSIONS}

The present paper has defined a new solution for the dispatching of the Energy Packets in a local DC grid. Based on previous researches on power/signal modulation applied to DC-DC power converters, the proposed research has demonstrated the applicability of one of these techniques, the PWM/PSK, for the dispatch of Energy packets among two or more nodes. This is a complete different vision than other ones, where multiple simultaneous messages or power/signal modulation were not considered. The simulations have shown the communication based on the PWM/PSK in a multiterminal DC grid where two different messages are simultaneously sent by two senders to the receivers, which are able to decode the correct messages addressed to them. This underpins the vision of the Energy Packet in analogy to packet-operation in the Internet.

However, there are still aspects that need to be further investigated that are related to the attenuation of the modulated signal in the electrical grid, the proper design of the digital filters and of the input/output filters of the converters and the definition of a range of switching frequencies of the converters that could be more suitable for the power/signal modulation.

Nevertheless the present paper has shown another solution in the direction of defining an electrical grid more and more similar to a computer network. The analogies between computer science and the energy domain shown throughout the paper demonstrate the necessity of creating a bridge between these two fields. Therefore, this is what the present research proposes: develop a common research that could make use of both competencies in order to define a complete revolutionary concept of the energy system.

\section{ACKNOWLEDGMENT}

The authors thank the E.ON ERC gGmbH for the financial support of the project "packET - packets for Energy Transformation". 


\section{REFERENCES}

[1] 2013. IEEE Standard for Low-Frequency (less than $500 \mathrm{kHz}$ ) Narrowband Power Line Communications for Smart Grid Applications. IEEE Std 1901.2-2013 (Dec 2013), 1-269. https://doi.org/10.1109/IEEESTD.2013.6679210

[2] Rikiya Abe, Hisao Taoka, and David McQuilkin. 2011. Digital grid: Communicative electrical grids of the future. IEEE Transactions on Smart Grid 2, 2 (2011), 399-410.

[3] John P Barton and David G Infield. 2004. Energy storage and its use with intermittent renewable energy. IEEE transactions on energy conversion 19,2 (2004), 441-448.

[4] KA Corzine. 2014. Energy packets enabling the energy internet. In Power Systems Conference (PSC), 2014 Clemson University. IEEE, 1-5.

[5] Jin D., Jiande Wu, Ruichi W., Zhengyu L., and Xiangning He. 2017. DC PowerLine Communication Based on Power/Signal Dual Modulation in Phase Shift Full-Bridge Converters. IEEE Transactions on Power Electronics 32, 1 (2017).

[6] Tomislav Dragicevic, Juan C Vasquez, Josep M Guerrero, and Davor Skrlec. 2014 Advanced LVDC Electrical Power Architectures and Microgrids: A step toward a new generation of power distribution networks. IEEE Electrification Magazine 2, 1 (2014), 54-65.

[7] Wei Jiang and Babak Fahimi. 2011. Multiport power electronic interfaceâĂŤconcept, modeling, and design. IEEE Transactions on Power Electronics 26, 7 (2011), 1890-1900.

[8] Prabha Kundur, Neal J Balu, and Mark G Lauby. 1994. Power system stability and control. Vol. 7. McGraw-hill New York.

[9] Lutz Lampe. 2016. Power Line Communications: Principles, Standards and Applications from Multimedia to Smart Grid. John Wiley \& Sons.

[10] A. Monti, E. De Din, D. MÃijller, F. Ponci, and V. Hagenmeyer. 2017. Towards a real digital power system: An energy packet approach. In 2017 IEEE Conference on Energy Internet and Energy System Integration (EI2). 1-6. https://doi.org/10. 1109/EI2.2017.8245741

[11] Paulo F Ribeiro, Brian K Johnson, Mariesa L Crow, Aysen Arsoy, and Yilu Liu. 2001. Energy storage systems for advanced power applications. Proc. IEEE 89, 12 (2001), 1744-1756.

[12] Ryo Takahashi, Shun-ichi Azuma, and Takashi Hikihara. 2016. Power regulation with predictive dynamic quantizer in power packet dispatching system. IEEE Transactions on Industrial Electronics 63, 12 (2016), 7653-7661.

[13] Ryo Takahashi, Keiji Tashiro, and Takashi Hikihara. 2015. Router for power packet distribution network: Design and experimental verification. IEEE Transactions on Smart Grid 6, 2 (2015), 618-626.

[14] A. Tanenbaum. 2002. Computer networks. Prentice Hall Professional Technical Reference.

[15] Ruichi Wang, Zhengyu Lin, Jin Du, Jiande Wu, and Xiangning He. 2017. Direct Sequence Spread Spectrum-Based PWM Strategy for Harmonic Reduction and Communication. IEEE Transactions on Power Electronics 32, 6 (2017), 4455-4465.

[16] J. Wu, J. Du, Z. Lin, Y. Hu, Chongwen Zhao, and Xiangning He. 2015. Power conversion and signal transmission integration method based on dual modulation of DC-DC converters. IEEE Transactions on Industrial Electronics 62, 2 (2015) 